\title{
Design to reliability shielded vertical interconnection applied to microwave Chip Scale Packaging
}

\author{
P. MONFRAIX ${ }^{(1)}$, T. ADAM $^{(1)}$, J. L. LACO STE ${ }^{(1)}$, C. DREVO N ${ }^{(1)}$, \\ G. NAUDY(1) ${ }^{(1)}$ B. $C O G O^{(1)}$, J.L. CAZAUX ${ }^{(1)}$, J.L. RO UX ${ }^{(2)}$
}

${ }^{(1)}$ ALCATEL SPACE INDUSTRIES, 26 avenue J .F. CHAMPO LLIO N

31037 TO ULO USE CEDEX, France

Tel : + 33 (0)5 343560 70, Fax : + 33 (0)5 3435 69-47

E-mail : Philippe.Monfraix@space.alcatel.fr

${ }^{(2)}$ CNES, 18 avenue E. BELIN, 31055 TO ULO USE CEDEX, France

\begin{abstract}
This paper presents the electrical design, measurement and reliability tests of a shielded vertical interconnection dedicated to microwave solder-mount packages.

Electromagnetic simulations show very good results up to $20 \mathrm{GHz}$. Test samples have been designed and manufactured. Electrical results are in accordance with the simulations with insertion loss lower than $0.1 \mathrm{~dB}$ up to $20 \mathrm{GHz}$ for the proposed interconnection. Reliability tests of present no degradation of the after 500 thermal cycles in the $\left[-55^{\circ} \mathrm{C},+125^{\circ} \mathrm{C}\right]$ temperature range.
\end{abstract}

\section{Introduction}

New services such as e-business, telemedecine, tele-teaching, illustrated by the drastic spread of Internet usage is synonym for space segment with constellation of satellites connecting people around the Earth. Numerous programs, such as SkyBridge from ALCATEL SPACE, are proposed to overcome these needs in voice, video and high speed data transfer. So, low cost solutions have to be found to face with a huge requirement in term of MMIC packaging.
Conventional MMIC packages use epoxy mounting and ribbon bonding solutions ([1]). This is frequency limited due to the associated inductance and very cost expensive for lack of automation. We proposed in this paper a solder-mount MMIC package through the design and the characterisation of a full shielded vertical interconnection between a ceramic package and a multilayer board. The interconnection, based on $\mu B G A$ arena, could be used as a Chip Scale Packaging for MMIC components. After a presentation of the concept, we will show the simulation results and the design of the test vehicles developed to characterise the transition. Then, electrical results and reliability tests will assess the proposed package.

\section{Description of the proposed interconnection}

The concept is proposed on Figure 1 . It is a connection from a ceramic package to a multilayer board through small diameter balls (like a $\mu B G A$ structure) in a coaxial shape. The microstrip line from the ceramic package is connected to the ball through a via hole. The microwave line of the board is embedded in the 
substrate (stripline) and connected to the balls through a via hole also.

The via hole connected to the microstrip line in the ceramic package and the via hole connected to the stripline in the board are directly aligned with the ball. Finally, the proposed interconnection is completely shielded, which is a great improvement to comparable packages $([2,3])$. For compensating the mismatching of thermo-elastic coefficients (CTE) of these two materials, an underfiller can be dispensed between the board (CTE close to $20 \mathrm{ppm}$ ) and the ceramic substrate (CTE close to $4.7 \mathrm{ppm}$ ).

\section{Electrical simulation}

Electrical simulations of the vertical interconnection have been performed on HFSS, a finite element 3D electromagnetic simulator. Two different designs have been proposed : with and without underfiller. The results, as depicted on Figure 2, show a good electrical characteristic up to $20 \mathrm{G} \mathrm{Hz}$ in the two configurations.

\section{Design and fabrication of test samples}

For the ceramic package, we used standard design rules from the MCMCeramic manufacturer. The balls, made of Copper, are directly AgCu brased on the Aluminium Nitride substrate.

For the board, it is Teflon based material for layers and pre-pregs.

Solder paste has been dispensed on the board and the ceramic package has been pick-and-placed then bonded by solder reflow.

The entire module depicted in Figure 3 has been placed on a $U$ shaped aluminium structure with SMA connectors for the electrical characterisation. Dispensing of an underfiller has also been represented on Figure 3, showing the fillet between the package and the board.

\section{Electrical measurements}

The measurement were performed with a TRL calibration from $0.1 \mathrm{MHz}$ to 20 $\mathrm{GHz}$ in the SMA connectors axis. A test vehicle was designed with only a straight stripline onto the board (no ceramic package and no vertical interconnection) to characterise the test structure : no issue can be mentioned (cf. Figure 4). On Figure 5, we described the results with/without underfiller of the whole package from one connector to another. The return loss is below -14 dB. With comparison of the straight stripline test vehicle, the insertion loss of one vertical interconnection can be estimated lower to $0.1 \mathrm{~dB}$ up to $20 \mathrm{G} \mathrm{Hz}$.

\section{Reliability tests}

To evaluate the reliability of the proposed interconnection, up to 500 thermal cycles were performed within $\left[-55^{\circ} \mathrm{C},+125^{\circ} \mathrm{C}\right]$ temperature range. Without underfiller, the package showed a drastic increasing contact resistance after 200 cycles. With underfiller, the package showed no degradation of the contact resistance up to 500 cycles. Destructive analysis confirm that point with no fatigue observed at the ball junction.

\section{Conclusion \& perspectives}

We presented in this paper a completely shielded vertical interconnection. Electrical simulations have been proposed. Test vehicles have been manufactured and assembled for electrical validation showing with a return loss better than $-14 \mathrm{~dB}$. The insertion loss per interconnection is estimated lower to $0.1 \mathrm{~dB}$ up to 20 $\mathrm{GHz}$. Reliability tests showed the necessity of an underfiller dispensing with 500 successful thermal cycles 
within $\left[-55^{\circ} \mathrm{C},+125^{\circ} \mathrm{C}\right]$ temperature range. With a reduction of the pad on board connecting the ball, a drastic improvement of this interconnection can be reached with a severe reduction of the capacitance associated. Associated to a low cost encapsulation (glued lid, glob top, ...), a solder-mount package for MMIC can be proposed for space applications.

\section{References}

[1] : "Multilayer printed circuit boards at 12-14 GHz with MCMs and MMICs",
C. Drevon et al., 1999 Wireless Workshop proceedings, Gold Canyon, Arizona.

[2] : "Ball grid arrays : a DC to 31.5 $\mathrm{GHz}$ low cost packaging solution for microwave and mm-wave MMICs", M.P.R. Panicker et al.; Microwave Journal, January 1998 issue.

[3] : "Development of CSP for microwave/ millimetrewave applications", K. Ikeda et al., 1999 Wireless Workshop proceedings, Gold Canyon, Arizona.

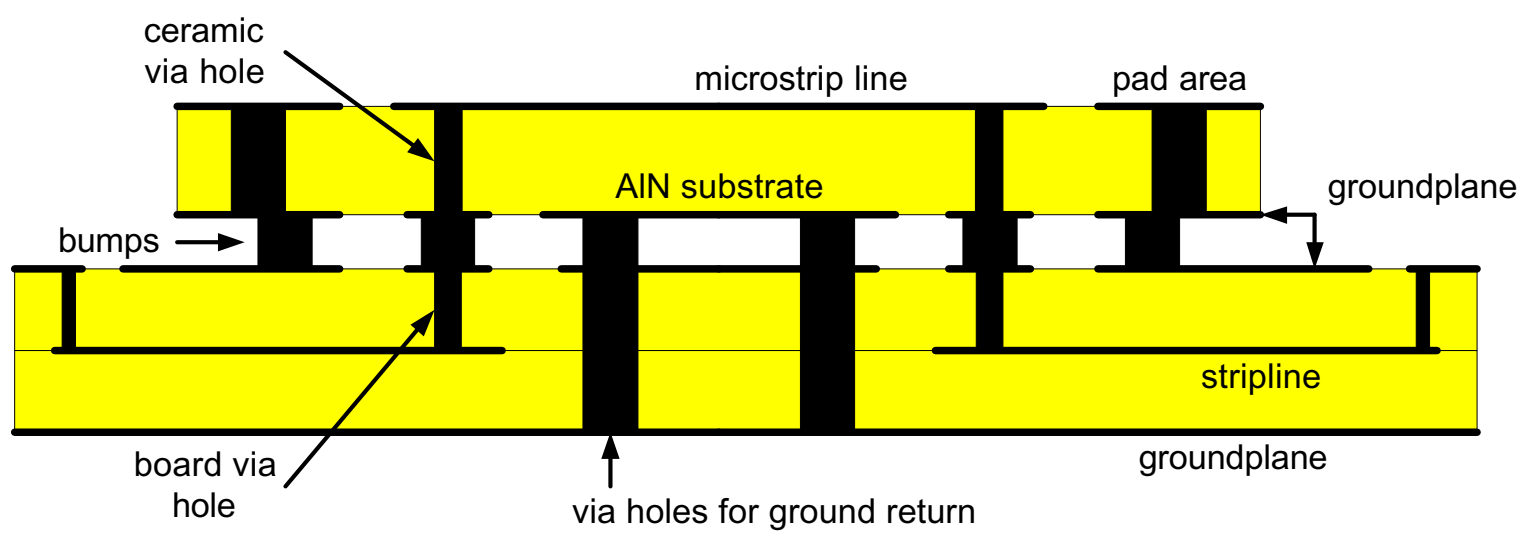

Figure 1 : Vertical Interconnect Packaging concept.

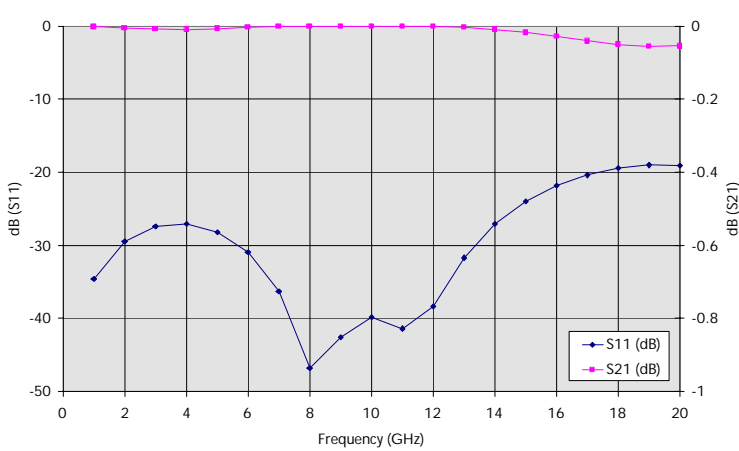

without underfiller

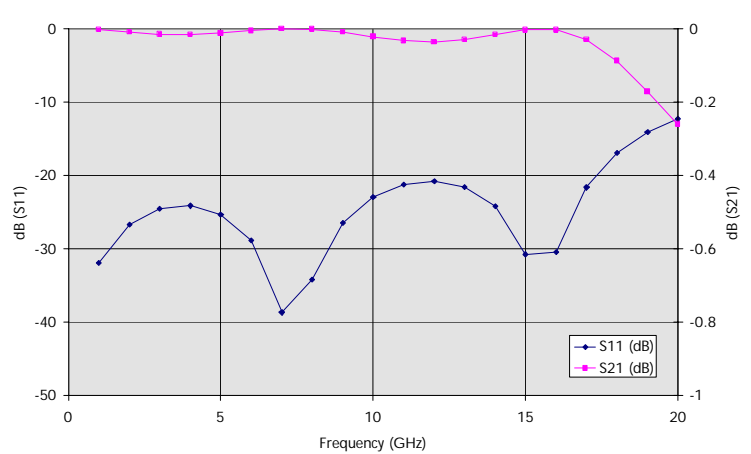

with underfiller

Figure 2 : Simulation results of the VIP interconnection. 


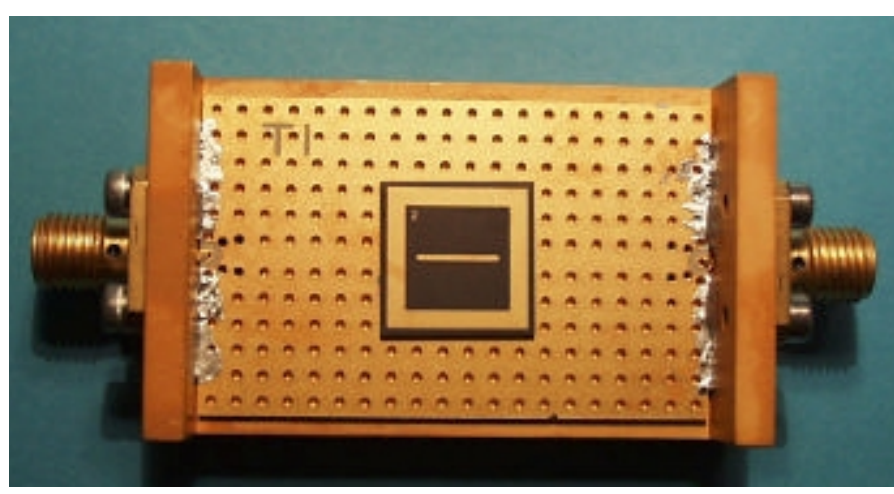

structure

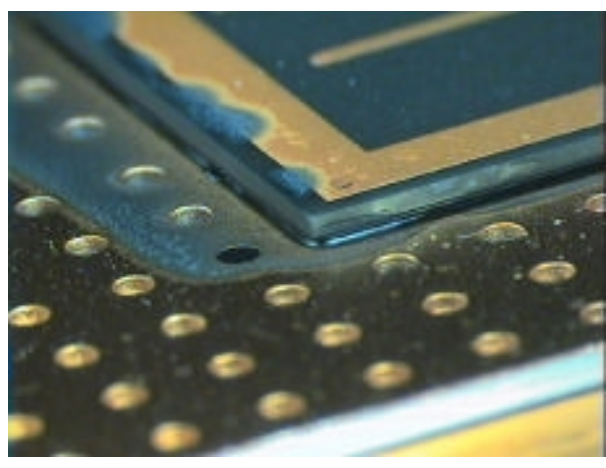

with underfiller

Figure 3 : Test vehide for the VIP electrical characterisation.

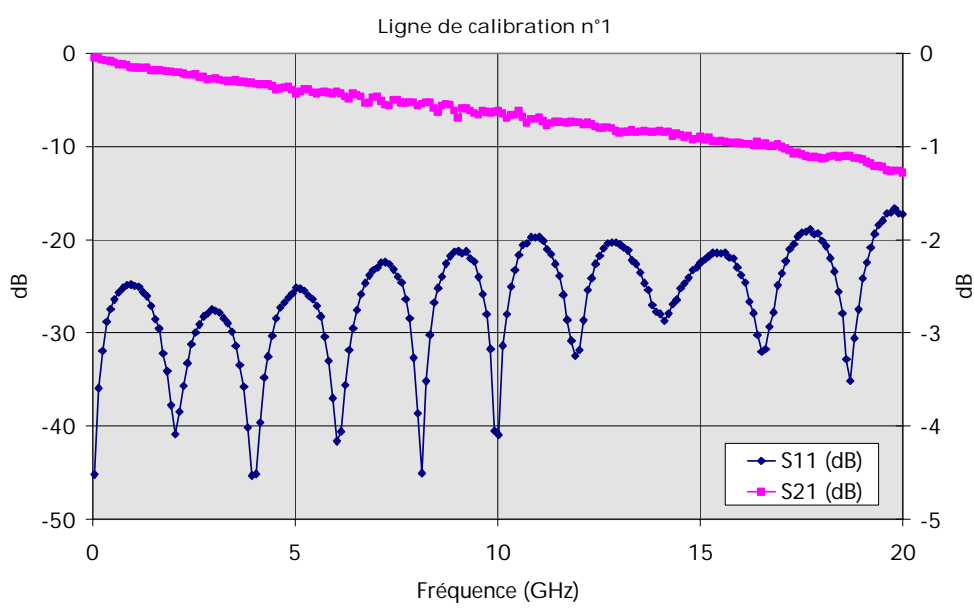

Figure 4 : Measurement of a thru stripline.

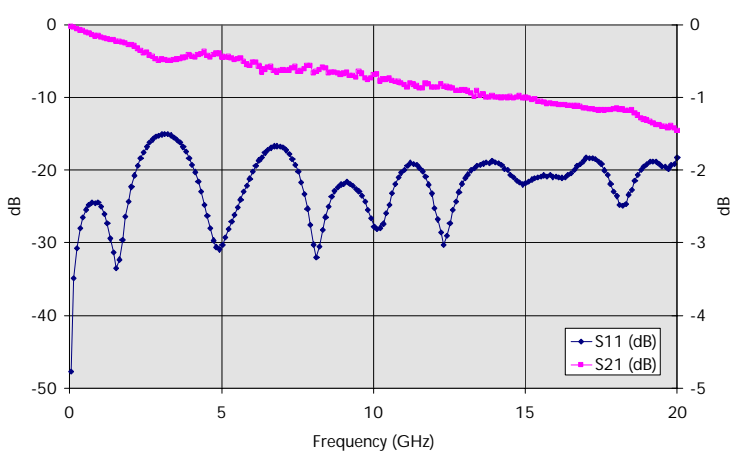

without underfiller

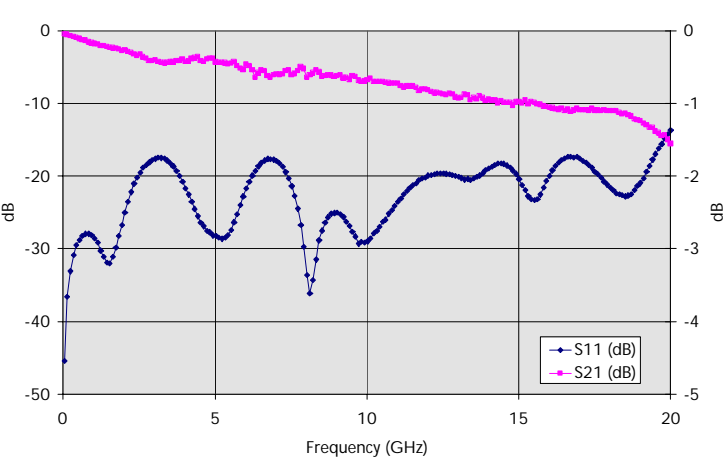

with underfiller

Figure 5 : Measurement of the test vehides with and without underfiller. 